\title{
Gleason 6 Prostate Cancer in One or Two Biopsy Cores Can Harbor More Aggressive Disease
}

\author{
Mark H. Katz, M.D., ${ }^{1,2}$ Sergey Shikanov, M.D., ${ }^{1}$ Maxine Sun, Ph.D., ${ }^{3}$ Firas Abdollah, M.D., ${ }^{3}$ \\ Lars Budaeus, M.D., ${ }^{3}$ Edward M. Gong, M.D.,' Scott E. Eggener, M.D.,', Gary D. Steinberg, M.D., \\ Gregory P. Zagaja, M.D., Arieh L. Shalhav, M.D., Pierre I. Karakiewicz, M.D., ${ }^{3}$ and Kevin C. Zorn, M.D.,3
}

\begin{abstract}
Background and Purpose: Patients with Gleason (GL) 6 prostate cancer in one or two biopsy cores can be upgraded and/or upstaged at the time of surgery, which may adversely impact long-term outcome. A novel model for prediction of adverse pathologic outcomes was developed using preoperative characteristics.

Patients and Methods: Between 2003 and 2007, 1159 patients underwent robot-assisted radical prostatectomy (RARP) at our institution. GL 6 prostate cancer in one or two biopsy cores was identified in 416 (36\%) patients. Logistic regression analyses were used to assess the rate of GL $\geq 7$ and/or extraprostatic extension at RARP. Covariates consisted of age, body mass index (BMI), number of positive cores, greatest percent of cancer in a core (GPC), clinical stage, and preoperative prostate-specific antigen (PSA) level. After backward variable selection, the developed model was internally validated using the area under the curve and subjected to methods of calibration.

Results: Respectively, 278 (67\%) and 138 (33\%) patients had one or two positive biopsy cores. At RARP, 90 (22\%) patients were upgraded to GL $\geq 7$ and $37(9 \%)$ had extraprostatic extension. The novel model relied on age, BMI, preoperative PSA level, and GPC for prediction of adverse pathologic outcomes and was 69\% accurate. Calibration plot revealed a virtually perfect relationship between predicted and observed probabilities.

Conclusions: In patients with GL 6 prostate cancer in one or two biopsy cores, $25 \%$ have more ominous pathology at RARP. The model provides an individual assessment of adverse outcomes at surgery. Consequently, it may be considered when counseling patients regarding their management options.
\end{abstract}

\section{Introduction}

$\mathbf{T}$ O BETTER ESTIMATE PROGNOSIS and tailor treatment options, much emphasis has been placed on risk stratification for prostate cancer. Even in the era of refined open surgical approaches, robot-assisted radical prostatectomy (RARP) and novel radiation delivery techniques all are associated with potential morbidity. Therefore, investigators have focused on identifying patients who can safely avoid treatment without significant risk for cancer progression and have evaluated the role of watchful waiting or active surveillance with selective delayed intervention. ${ }^{1}$

Patients with pathologically organ-confined, low-grade tumors (Gleason [GL] grade $\leq 6$ ) are most likely to manifest an indolent natural history and appear to be the most appropriate candidates for active surveillance strategies. ${ }^{2}$ Multiple reports, however, have found that $20 \%$ to $25 \%$ of low-volume, low-grade cancers on prostate biopsy are upgraded and/or upstaged at surgery. ${ }^{3,4}$ These findings suggest incomplete characterization of biopsy-detected lowvolume, low-grade prostate cancer and a compelling need for further investigation. In particular, identifying clinical variables that may predict upgrading and/or upstaging at surgery would be useful when counseling patients on their treatment options.

With a minimum of a 12-core prostate biopsy, patients with GL 6 prostate cancer in a small percentage of one or two cores are especially likely to be counseled on the potentially indolent nature of their disease. Based on the increasing number of men with low-volume, low-grade cancer on biopsy and the potential risk of more aggressive pathology at the time of surgery, we sought to develop a model that is able to predict the risk of adverse pathologic outcomes at surgery using preoperative variables.

\footnotetext{
${ }^{1}$ Section of Urology, Department of Surgery, University of Chicago Medical Center, Chicago, Illinois.

${ }^{2}$ Department of Urology, Boston University, Boston, Massachusetts.

${ }^{3}$ Section of Urology, Department of Surgery, University of Montreal Hospital Center, Montreal, Quebec, Canada.
} 


\section{Patients and Methods}

\section{Patients}

Between February 2003 and November 2007, 1159 patients in our RARP database approved by our Institutional Review Board approved were identified, of whom 416 (36\%) had GL 6 prostate cancer in one or two biopsy cores. All patients underwent ultrasonography-guided prostate biopsy, and the number of cores sampled was documented. No patients had neoadjuvant therapy or previous pelvic radiation. All surgeries were performed by three surgeons (KCZ, GPZ, and ALS). Our surgical technique for RARP has been described previously. ${ }^{5}$ Selected patients underwent bilateral pelvic lymph node dissection (PLND) at the discretion of the primary surgeon-typically those with prostate-specific antigen (PSA) level $>10 \mathrm{ng} / \mathrm{mL}$, primary GL pattern $\geq 4$, or palpable disease.

\section{Pathologic analysis}

Although most prostate biopsies were performed elsewhere, all specimens were reviewed by a dedicated uropathologist at our institution. RARP specimens were processed according to a modified Stanford protocol. ${ }^{6}$ Briefly, the specimen is fixed overnight in $10 \%$ formalin and its external surface inked. The apical and bladder neck margins are shaved off, serially sectioned, and entirely submitted for histologic processing. The rest of the gland is transversely sectioned at 3-mm intervals (approximately 6-12 sections per gland). Sections comprising approximately $70 \%$ of a 50 -g prostatectomy specimen, including $90 \%$ of the peripheral zone, are submitted for histologic processing. The sections are then cut at $5 \mu \mathrm{m}$, stained with hematoxylin and eosin, and examined with light microscopy by the same pathologist who analyzed the prostate biopsy specimens.

\section{Description of covariates}

Preoperative characteristics included patient age (years), body mass index (BMI), preoperative PSA level, number of positive cores for cancer (dichotomized as one vs two), greatest percent of cancer in a core (GPC), and clinical stage $\left(\mathrm{T}_{1 \mathrm{c}}\right.$ vs $\left.\mathrm{T}_{2}\right)$. Pathologic information included grade, stage, and surgical margin status. Adverse pathology was defined as GL $\geq 7$ and/or pathologic stage $\geq \mathrm{pT}_{3}$.

\section{Statistical analysis}

Descriptive characteristics for continuously coded variables were represented using means and medians $v s$ frequencies and proportions for categorically coded variables. To assess whether the predictive ability of continuously coded variables may be improved, we introduced cutoffs using the minimum $P$-value approach, as described by Mazumdar and Glassman. ${ }^{7}$ Univariable and multivariable logistic regression analyses were fitted for prediction of adverse pathologic outcomes at $\operatorname{RARP}\left(\mathrm{GL} \geq 7\right.$ and $/$ or $\geq \mathrm{pT}_{3}$ ). All preoperative variables were considered.

Subsequently, a backward selection process was applied, which uses the Akaike information criteria as a stopping rule to identify the most informative variables. The accuracy of the model relying on the most informative predictors of adverse pathologic outcomes was quantified using the receiver oper- ating characteristics-derived area under the curve. A value of $50 \%$ is equivalent to a toss of a coin and $100 \%$ represents a perfect discriminatory ability. Finally, the relationship between predicted and observed probabilities was graphically depicted using calibration plot.

All statistical analysis was performed using JMP 7 statistical software (SAS Institute; Cary, NC), the R Foundation for Statistical Computing, and Predictive Analytics Software, ${ }^{\circledR}$ version 18.0. Moreover, all tests were two-sided with a significance level set at $P<0.05$.

\section{Results}

The mean (range) patient age, BMI, and preoperative PSA level was 59 years (median $60 \mathrm{y}$ ), $28.1 \mathrm{~kg} / \mathrm{m}^{2}$ (median $27.6 \mathrm{~kg} / \mathrm{m}^{2}$ ), and $5.9 \mathrm{ng} / \mathrm{mL}$ (median $35.0 \mathrm{ng} / \mathrm{mL}$ ), respectively (Table 1). Respectively, $372(89 \%)$ and $44(11 \%)$ were clinical stage $\mathrm{T}_{1 \mathrm{c}}$ and $\mathrm{T}_{2}$. The mean (range) number of biopsy cores sampled was 12 (median 12), where 133 (31\%) patients had $<12$ cores. Respectively, 278 (67\%) and $138(33 \%)$ patients had one or two biopsy cores with GL 6 prostate cancer.

A total of $107(25 \%)$ patients had adverse pathology at RARP: 90 (22\%) were upgraded to GL $\geq 7$ (three of which were GL 8), and $37(9 \%)$ were upstaged to $\geq \mathrm{pT}_{3}$ (Table 2). PLND was performed in $35(8 \%)$ patients, all negative for cancer. In addition, $56(14 \%)$ patients demonstrated positive surgical margins. With a mean follow-up of 15 months, there were 18 ( $4 \%$ ) biochemical recurrences (PSA $\geq 0.2 \mathrm{ng} / \mathrm{mL}$ and rising).

The ability of all baseline patient characteristics in predicting adverse pathologic outcomes was tested. For continuously coded variables, cutoffs were systematically assessed to determine whether categorization might improve predictive ability. These were introduced according to the $P$-value approach and are summarized in Table 3. This method yielded informative cutoffs for all continuously coded variables.

Table 1. Clinical Characteristics of the Study Population $(\mathrm{N}=416)$

$\begin{array}{lc}\text { Variables } & \\ \text { Number of patients } & 416 \\ \text { Age (years) } & \\ \quad \text { Mean (median) } & 59.2(60.0) \\ \quad \text { Range } & 40-77 \\ \text { Body mass index }\left(\mathrm{kg} / \mathrm{m}^{2}\right) & 28.1(27.6) \\ \quad \text { Mean (median) } & 1.8-5.9 \\ \quad \text { Range } & \\ \text { Prostate-specific antigen }(\mathrm{ng} / \mathrm{mL}) & 5.9(5.0) \\ \quad \text { Mean (median) } & 0.6-35.0 \\ \quad \text { Range } & 12.0(12.0) \\ \text { Number of cores sampled } & 6-40 \\ \quad \text { Mean (median) } & \\ \quad \text { Range } & 278(66.8) \\ \text { Number of positive cores } & 138(33.2) \\ \quad 1 & \\ 2 & 372(89.4) \\ \text { Clinical stage } & 44(10.6) \\ \quad \mathrm{T}_{1 \mathrm{c}} & 12.0(5.0) \\ \quad \mathrm{T}_{2} & 1-90 \\ \text { Greatest percent of cancer in a core } & \\ \quad \text { Mean (median) } & \\ \text { Range } & \end{array}$


Table 2. Pathologic Outcomes of the Study Population

\begin{tabular}{lr}
\hline Variables & \\
Pathologic tumor stage & $379(91.1)$ \\
$\mathrm{pT}_{2}$ & $34(8.2)$ \\
$\mathrm{pT}_{3 \mathrm{a}}$ & $3(0.7)$ \\
$\mathrm{pT}_{3 \mathrm{~b}}$ & $326(78.4)$ \\
Pathologic Gleason grade & $87(20.9)$ \\
6 & 75 \\
7 & 12 \\
$3+4=7$ & $3(0.7)$ \\
$4+3=7$ & $107(25 \%)$ \\
$\geq 8$ & $14(13.1)$ \\
Upstaged/upgraded $\left(\mathrm{GL} \geq 7 / \geq \mathrm{pT}_{3}\right)$ & $13(12.1)$ \\
Total number of patients & $56(13.5)$ \\
$\bullet \% \mathrm{PSA}>10$ ng/Ml & \\
- \%cT & \\
Positive surgical margins & \\
PLND & \\
Patients with PLND & \\
Patients with lymph node involvement &
\end{tabular}

In consequence, only categorically coded variables were used within subsequent logistic regression models.

Univariable logistic regression analyses (Table 3) demonstrated that patients with GPC $>10$ (odds ratio [OR]: 3.3, $P<0.001$ ), age $>66$ (OR: $2.2, P=0.01$ ), $\mathrm{BMI}>38.4$ (OR: 4.1 , $P=0.01$ ), and PSA level $>8.7 \mathrm{ng} / \mathrm{mL}(\mathrm{OR}: 2.1, P=0.0)$ were significantly associated with a higher rate of adverse pathologic outcome at RARP. Conversely, clinical stage and the number of positive biopsy cores failed to achieve statistical significance. Similar findings were revealed in multivariable analyses when all covariates were considered (Table 4). After backward variable selection, only age, BMI, preoperative PSA level, and GPC remained in the model (Fig. 1A). The predictive accuracy of the final model was $69 \%$. The calibration plot demonstrated virtually no departures from ideal predictions (Fig. 1B).

Table 3. Descriptives of Patient Characteristics According to the Most Significant Cutoffs for Continuously Coded Variables

\begin{tabular}{lcc}
\hline Variables & Number of patients (\%) & P value \\
\hline $\begin{array}{c}\text { Age (years) } \\
\leq 66\end{array}$ & $367(88.2)$ & \\
$>66$ & $49(11.8)$ & 0.02 \\
BMI & & \\
$\leq 38.41$ & $402(96.6)$ & 0.02 \\
$>38.41$ & $14(3.4)$ & \\
Preoperative PSA level & & \\
$\leq 8.7$ & $360(86.5)$ & 0.02 \\
$>8.7$ & $56(13.5)$ & \\
GPC (\%) & & \\
$\leq 10$ & $310(74.5)$ & \\
$>10$ & $106(25.5)$ & \\
\hline
\end{tabular}

$\mathrm{BMI}=$ body mass index; $\mathrm{PSA}=$ prostate-specific antigen; $\mathrm{GPC}=$ greatest percent of cancer in a core.

\section{Discussion}

Men with GL 6 prostate cancer in one or two biopsy cores represent more than one-third of our institutional RARP database and have a $25 \%$ risk of harboring more adverse pathologic features at the time of surgery. Patients can be more accurately risk-stratified using the novel model based on age, BMI, preoperative PSA level, and GPC.

Because of sampling error of ultrasonography-guided prostate biopsy, the phenomenon of upgrading at the time of radical prostatectomy has been well documented. The current study reveals that $21 \%$ of patients are upgraded to GL $\geq 7$, comparable to Gofrit and associates ${ }^{4}$ reporting that $20 \%$ of patients with GL 6 disease on biopsy were upgraded. Another contemporary study by Griffin and colleagues ${ }^{3}$ analyzed the outcomes of patients with GL 6 cancer in one or two biopsy cores who underwent open radical prostatectomy and found that $27 \%$ of patients were upgraded. Other series, however, have reported higher rates of GL 6 upgrading, ranging from $37 \%$ to $62 \%$. $^{8-14}$

We believe that the higher rates of biopsy and surgical pathology concordance in our series and the study by Griffin and coworkers ${ }^{3}$ reflect contemporary biopsy and pathologic grading practices. More than two-thirds of the patients in our study were more likely to undergo extensive biopsy sampling with at least $12(69 \%)$ cores, minimizing the likelihood of upgrading because of sampling error. In addition, reevaluation of all biopsy specimens at our institution by dedicated uropathologists may have decreased the rate of upgrading.

The upstaging of clinically localized prostate cancer has also been documented in the literature. Bastian and colleagues $^{14}$ analyzed 237 men who underwent radical prostatectomy for clinically insignificant prostate cancer as defined by the Epstein criteria (clinical stage $\mathrm{T}_{1 \mathrm{c}}$, PSA density $<0.15$, GL score $\leq 6,<3$ positive cores, and $\leq 50 \%$ involvement of any core). Extraprostatic extension was identified in $8.4 \%$, a finding very similar to the $10 \%$ of patients in our study who were upstaged.

Our study found that a GPC of $>10 \%$ was predictive of adverse pathology. GPC has proven to be a surrogate marker for tumor volume in another study. ${ }^{15}$ Age $>65$ years also proved to have prognostic significance for upgrading or upstaging. This may be especially important when counseling healthy older men on their management options for lowvolume GL 6 prostate cancer. Many clinicians are reluctant to offer surgery to older patients with these biopsy findings, but our data suggest a significant risk for harboring more aggressive disease in this cohort. Similar to previous studies, higher preoperative PSA levels $(>10 \mathrm{ng} / \mathrm{mL})$ in the setting of low-volume GL 6 disease still proved to be prognostic for adverse pathology. ${ }^{16}$

The number of positive cores (one vs two) and clinical stage were not significantly affected with the risk of upgrading or upstaging. Previous studies with more equivalent proportions of $T_{1 c}$ and $T_{2}$ patients have shown clinical stage to be a predictor of GL upgrading. ${ }^{10}$ However, $89 \%$ of our cohort was $\mathrm{T}_{1 \mathrm{c}}$, a finding that reflects contemporary practice.

While controversy still persists over how to best manage low-volume GL 6 prostate cancer, our data reiterate that many of these patients harbor clinically significant cancer that may warrant early intervention. Until advances are made in molecular markers, imaging, or other methods of optimizing 
Table 4. Univariable and Multivariable Logistic Regression Analyses for Prediction of Adverse Pathologic Outcomes at Surgery

\begin{tabular}{|c|c|c|c|c|c|c|}
\hline \multirow[b]{2}{*}{ Variables } & \multirow[b]{2}{*}{ Univariable OR, 95\% CI } & \multirow[b]{2}{*}{$\mathrm{P}$} & Full model & \multirow[b]{2}{*}{$\mathrm{P}$} & Reduced model & \multirow[b]{2}{*}{$\mathrm{P}$} \\
\hline & & & Multivariable OR, 95\% CI & & Multivariable OR, 95\% CI & \\
\hline \multicolumn{7}{|l|}{ GPC } \\
\hline$\leq 10$ & 1.0 (ref.) & \multirow[t]{2}{*}{$<0.001$} & 1.0 (ref.) & \multirow[t]{2}{*}{$<0.001$} & 1.0 (ref.) & \multirow[t]{2}{*}{$<0.001$} \\
\hline$>10$ & $3.32(2.06-5.34)$ & & $3.08(1.87-5.09)$ & & $3.17(1.94-5.19)$ & \\
\hline \multicolumn{7}{|l|}{ PSA } \\
\hline$\leq 8.7$ & 1.0 (ref.) & \multirow[t]{2}{*}{0.01} & 1.0 (ref.) & \multirow[t]{2}{*}{0.02} & 1.0 (ref.) & \multirow[t]{2}{*}{0.02} \\
\hline$>8.7$ & $2.09(1.16-3.77)$ & & $2.15(1.15-4.05)$ & & $2.10(1.12-3.93)$ & \\
\hline \multicolumn{7}{|l|}{ BMI } \\
\hline$\leq 38.41$ & 1.0 (ref.) & \multirow[t]{2}{*}{0.01} & 1.0 (ref.) & \multirow[t]{2}{*}{0.09} & 1.0 (ref.) & \multirow[t]{2}{*}{0.07} \\
\hline$>38.41$ & $4.08(1.38-12.05)$ & & $2.74(0.84-8.91)$ & & $2.95(0.91-9.59)$ & \\
\hline \multicolumn{6}{|l|}{ Age } & \multirow{3}{*}{0.04} \\
\hline$\leq 66$ & 1.0 (ref.) & \multirow[t]{2}{*}{0.01} & 1.0 (ref.) & \multirow[t]{2}{*}{0.03} & 1.0 (ref.) & \\
\hline$>66$ & $2.20(1.20-4.12)$ & & $2.02(1.05-3.88)$ & & $2.00(1.04-3.83)$ & \\
\hline \multicolumn{7}{|c|}{ Number of positive cores } \\
\hline 1 & 1.0 (ref.) & \multirow[t]{2}{*}{0.02} & 1.0 (ref.) & \multirow[t]{2}{*}{0.1} & - & \multirow[t]{2}{*}{ 一 } \\
\hline 2 & 1.69 (1.07-2.66) & & $1.50(0.93-2.43)$ & & & \\
\hline \multicolumn{7}{|c|}{ Clinical stage } \\
\hline $\mathrm{cT}_{1 \mathrm{c}}$ & 1.0 (ref.) & \multirow[t]{2}{*}{0.5} & 1.0 (ref.) & \multirow[t]{2}{*}{0.7} & - & \multirow[t]{2}{*}{ - } \\
\hline $\mathrm{cT}_{2}$ & $1.24(0.62-2.47)$ & & $0.88(0.42-1.96)$ & & & \\
\hline
\end{tabular}

$\mathrm{OR}=$ odds ratio; $\mathrm{CI}=$ confidence interval; $\mathrm{GPC}=$ greatest percent of cancer in a core; ref. = referent category; PSA $=$ prostate-specific antigen; $\mathrm{BMI}=$ body mass index.

disease characterization, the foundation of risk stratification remains the preoperative clinical variables. Counseling lowrisk patients on their risk of harboring more aggressive disease is difficult. Our findings suggest, however, that age, PSA level, and GPC are particularly important parameters to discuss with patients.

There are several limitations of the present study that warrant mention. Our study is a single institution experience, and collaborative studies with other high-volume centers are necessary to corroborate the findings. Given the retrospective nature of the study and that all patients underwent RARP as opposed to active surveillance or other treatment modalities, there is undoubtedly a selection bias. Because the patient population is contemporary with relatively short follow-up, we have limited our analysis to pathologic outcomes. Upgrading and upstaging are clearly adverse pathologic findings, but they do not necessarily translate into worse clinical outcomes. Longer follow-up is necessary to evaluate cancerspecific and overall survival. All biopsy specimens were internally reviewed; however, the majority of biopsy procedures
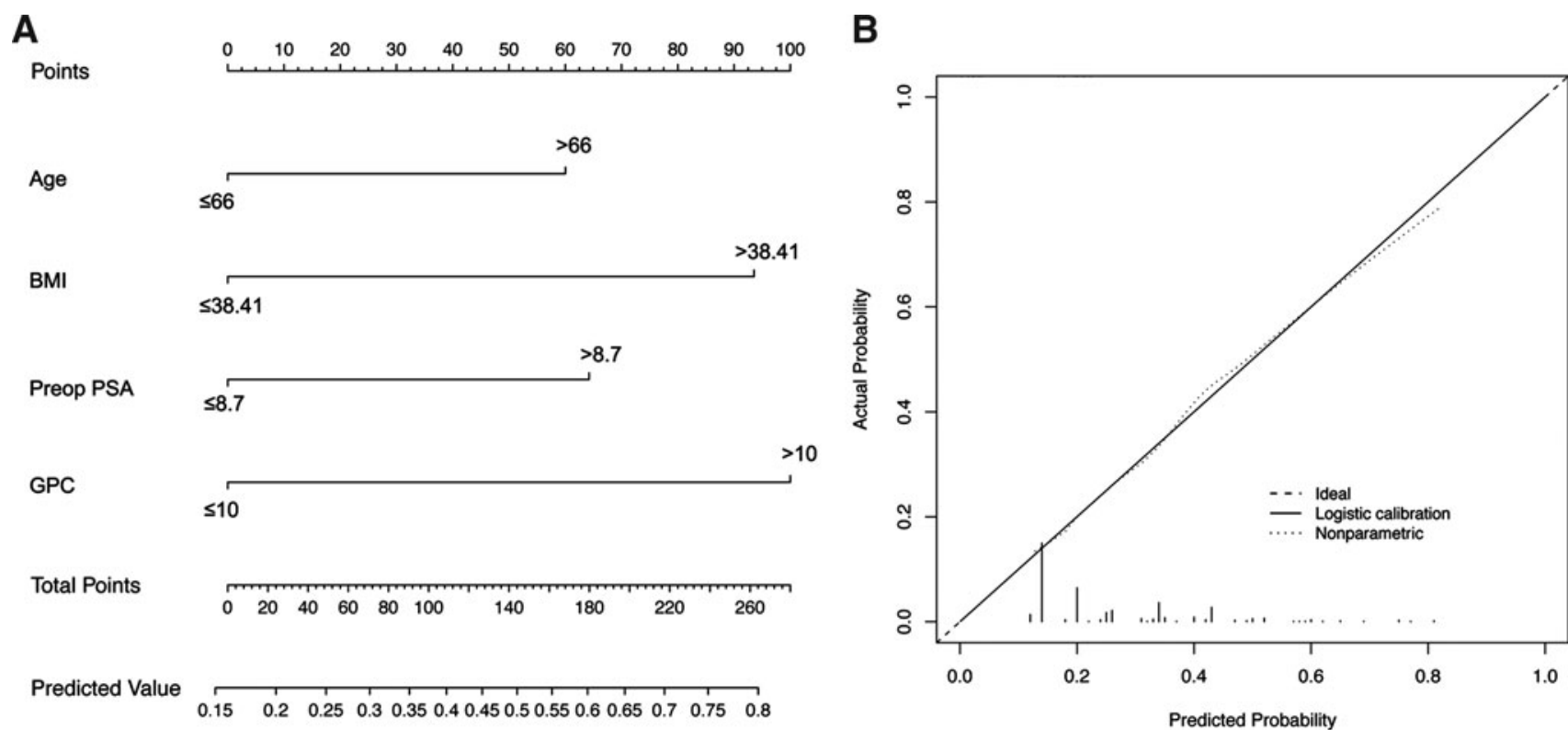

FIG. 1. (A) Nomogram predicting adverse pathological outcomes of men with Gleason 6 in 1-2 cores on prostate biopsy. (B) Calibration plot of nomogram in internal validation cohort. BMI = body mass index; PSA= prostate-specific antigen; $\mathrm{GPC}=$ greatest percent of cancer in a core . 
were performed elsewhere, making it difficult to control for consistency of technique or number of cores sampled. Finally, we selected patients with GL 6 prostate cancer in one or two cores as our study population, because they are most likely to consider active surveillance as a management option. Many different inclusion criteria are used by clinicians, however, when considering candidates for active surveillance. Using alternative definitions of low-risk disease would likely lead to different rates of adverse pathology at the time of surgery.

\section{Conclusion}

In a contemporary RARP series of patients with GL 6 prostate cancer in one or two biopsy cores, $25 \%$ were upgraded to GL $\geq 7$ or upstaged to extraprostatic disease on final pathology findings. GPC $>15 \%$, age $>65$, and PSA level $>10 \mathrm{ng} / \mathrm{mL}$ were predictive of adverse pathology. These findings suggest that many patients who are considered to have indolent disease may harbor more aggressive pathology. This information is helpful for patients who are considering immediate treatment $v s$ active surveillance.

\section{Disclosure Statement}

No competing financial interests exist.

\section{References}

1. Eggener SE, Mueller A, Berglund RK, et al. A multiinstitutional evaluation of active surveillance for low risk prostate cancer. J Urol 2009;181:1635-1641.

2. Albertson PC, Hanley JA, Fine J. 20-year outcomes following conservative management of clinically localized prostate cancer. JAMA 2005;293:2095-2101.

3. Griffin CR, Yu X, Loeb S, et al. Pathological features after radical prostatectomy in potential candidates for active monitoring. J Urol 2007;178:860-863.

4. Gofrit ON, Zorn KC, Taxy JB, et al. Predicting the risk of patients with biopsy Gleason score 6 to harbor a higher grade cancer. J Urol 2007;178:1925-1928.

5. Zorn KC, Gofrit ON, Orvieto MA, et al. Robotic-assisted laparoscopic prostatectomy: Functional and pathologic outcomes with interfascial nerve preservation. Eur Urol 2007;51: 755-63.

6. Amin MB, Grignon D, Bostwick D, et al. Recommendations for the reporting of resected prostate carcinomas: Association of Directors of Anatomic and Surgical Pathology. Am J Clin Pathol 1996;105:667-670.

7. Mazumdar M, Glassman JR. Categorizing a prognostic variable: Review of methods, code for easy implementation and applications to decision-making about cancer treatments. Stat Med 2000:19;113-132.
8. Steinberg DM, Sauvageot J, Piantadosi S, Epstein JI. Correlation of prostate needle biopsy and radical prostatectomy Gleason grade in academic and community settings. Am J Surg Pathol 1997;21:566-576.

9. Cookson MS, Fleshner NE, Soloway SM, Fair WR. Correlation between Gleason score of needle biopsy and radical prostatectomy specimen: Accuracy and clinical implications. J Urol 1997;157:559-562.

10. Chun FK, Briganti A, Graefen M, et al. Development and external validation of an extended 10-core biopsy nomogram. J Urol 2007;177:510-515.

11. King C. Patterns of prostate cancer biopsy grading: Trends and clinical implications. Int J Cancer 2000;90:305-311.

12. Pinthus JH, Witkos M, Fleshner NE, et al. Prostate cancers scored as Gleason 6 on prostate biopsy are frequently Gleason 7 tumors at radical prostatectomy: Implication on outcome. J Urol 2006;176:979-984.

13. Fukagai T, Namiki T, Namiki H, et al. Discrepancies between Gleason scores of needle biopsy and radical prostatectomy specimens. Pathol Int 2001;51:364-370.

14. Bastian PJ, Mangold LA, Epstein JI, Partin AW. Characteristics of insignificant clinical T1c prostate tumors. A contemporary analysis. Cancer 2004;101:2001-2005.

15. Epstein JI, Walsh PC, Carmichael M, Brendler CB. Pathologic and clinical findings to predict tumor extent of nonpalpable (stage T1c) prostate cancer. JAMA 1994;271:368374.

16. Sebo TJ, Bock BJ, Cheville JC, et al. The percent of cores positive for cancer in prostate needle biopsy specimens is strongly predictive of tumor stage and volume at radical prostatectomy. J Urol 2000;163:174-178.

Address correspondence to: Kevin C. Zorn, M.D. Section of Urology University of Montreal Hospital Center 235 boul Rene Levesque East, Suite 301 Montreal, QC, H2X 1N8 Canadá

E-mail: kevin.zorn@gmail.com

$$
\begin{aligned}
& \text { Abbreviations Used } \\
\mathrm{BMI} & =\text { body mass index } \\
\mathrm{GL} & =\text { Gleason } \\
\mathrm{GPC} & =\text { greatest percent of cancer in a core } \\
\mathrm{PLND} & =\text { pelvic lymph node dissection } \\
\mathrm{PSA} & =\text { prostate-specific antigen } \\
\mathrm{RARP} & =\text { robot-assisted radical prostatectomy }
\end{aligned}
$$




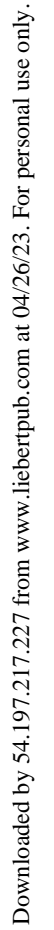

